\title{
On the estimate of the fourth-order homogeneous coefficient functional for univalent functions
}

\author{
by Larisa Gromova and Alexander Vasil'ev (Saratov)
}

\begin{abstract}
The functional $\left|c_{4}+p c_{2} c_{3}+q c_{2}^{3}\right|$ is considered in the class $\mathbb{S}$ of all univalent holomorphic functions $f(z)=z+\sum_{n=2}^{\infty} c_{n} z^{n}$ in the unit disk. For real values $p$ and $q$ in some regions of the $(p, q)$-plane the estimates of this functional are obtained by the area method for univalent functions. Some new regions are found where the Koebe function is extremal.
\end{abstract}

Introduction. Let $\mathbb{S}$ be the class of all holomorphic univalent functions

$$
f(z)=z+\sum_{n=2}^{\infty} c_{n} z^{n}
$$

in the unit disk. We consider the functional

$$
D_{4}(f)=c_{4}+p c_{2} c_{3}+q c_{2}^{3}
$$

for real values $p$ and $q$ which is fourth-order homogeneous in the sense of rotation:

$$
e^{-3 i \alpha} D_{4}\left(e^{-i \alpha} f\left(e^{i \alpha} z\right)\right)=D_{4}(f)
$$

Many papers are devoted to the estimation of $\left|D_{4}\right|$ for different values of $p$ and $q$ (see [1], [2], [4], [5]). Special interest in this functional is connected with estimating the seventh coefficient $c_{7}^{(2)}$ in the class $S^{(2)}$ of odd univalent functions

$$
f(z)=z+\sum_{n=2}^{\infty} c_{2 n-1}^{(2)} z^{2 n-1}
$$

1991 Mathematics Subject Classification: Primary 30C55.

Key words and phrases: univalent function, area method.

This research is supported by Russian Foundation of Basic Research, grant N 95-01$00345 \mathrm{~A}$. 
Namely, $\max _{f \in S^{(2)}}\left|c_{7}^{(2)}\right|=\max _{f \in \mathbb{S}} 2^{-1}\left|D_{4}(f)\right|$ for $p=-1 / 2, q=1 / 8$. P. Lehto [4] showed that $\left|D_{4}\right| \leq 4+6 p+8 q$ when $q \geq p^{2} / 4+p / 4+7 / 12$, with the Koebe function being extremal. Moreover, he found that if $p=-2$ and $q=13 / 12$, then the Koebe function is not unique.

Here we find some other regions in the $(p, q)$-plane where the Koebe function is extremal and find new regions where the estimates are different from $4+6 p+8 q$.

We use the area method in the form given by N. Lebedev [3] for the estimate of the fourth coefficient for univalent functions.

Let $F^{(2)}(\zeta)$ belong to the class $\Sigma^{(2)}$ of all odd univalent functions $F(\zeta)=$ $\zeta+a_{1} / \zeta+a_{3} / \zeta^{3}+\ldots$ in the exterior of the unit disk $|\zeta|>1$. Then

$$
\operatorname{Ln} \frac{F^{(2)}(\zeta)-F^{(2)}(t)}{\zeta-t}=\sum_{n, m=1}^{\infty} \omega_{n m} \zeta^{-n} t^{-m}, \quad|t|>1,
$$

where $\omega_{p q}$ are the Grunsky coefficients. It is known [3] that

$$
\left\{\begin{array}{l}
c_{4}=2 \omega_{33}+8 \omega_{11} \omega_{13}+\frac{10}{3}\left(\omega_{11}\right)^{3} \\
c_{3}=2 \omega_{13}+3\left(\omega_{11}\right)^{2} \\
c_{2}=2 \omega_{11}
\end{array}\right.
$$

By the Grunsky inequality for any $l \in \mathbb{C}$ we get

$$
\left|\omega_{33}+2 \omega_{13} l+\omega_{11} l^{2}\right| \leq\left|l^{2}\right|+1 / 3,
$$

and from (1),

$$
D_{4}=c_{4}+p c_{2} c_{3}+q c_{2}^{3}=2 \omega_{33}+4(2+p) \omega_{11} \omega_{13}+2(5 / 3+4 q+3 p) \omega_{11}^{3}
$$

and

(2) $\quad \mid D_{4}-4(2+p) \omega_{11} \omega_{13}-2(5 / 3+4 q+3 p) \omega_{11}^{3}$

$$
+4 \omega_{13} l+\left.2 \omega_{11} l^{2}|\leq 2| l\right|^{2}+2 / 3 .
$$

For convenience we assume $\omega_{13}=\omega_{3}$ and $\omega_{11}=\omega_{1}$. Since $D_{4}(f)=$ $e^{-3 i \alpha} D_{4}\left(e^{-i \alpha} f\left(e^{i \alpha} z\right)\right)$, we assume $D_{4} \geq 0$ without loss of generality. The modulus on the left-hand side of (2) can be replaced by the real part, so

$$
\begin{gathered}
\operatorname{Re} D_{4} \leq 2 / 3+2|l|^{2}+\operatorname{Re}\left\{4\left((2+p) \omega_{1}-l\right) \omega_{3}\right. \\
\left.+2(5 / 3+4 q+3 p) \omega_{1}^{3}-2 \omega_{1} l^{2}\right\} .
\end{gathered}
$$

The area theorem for odd univalent functions [3] states that

$$
\sum_{n=1}^{\infty}(2 n-1)\left|\omega_{1,2 n-1}\right|^{2} \leq 1
$$


Therefore $\left|\omega_{1}\right|^{2}+3\left|\omega_{3}\right|^{2} \leq 1$ or $\left|\omega_{3}\right| \leq(1 / \sqrt{3}) \sqrt{1-\left|\omega_{1}\right|^{2}}$. Thus

$$
\begin{aligned}
\left|D_{4}\right| \leq & 2 / 3+2|l|^{2}+\left|4\left((2+p) \omega_{1}-l\right)\right| \frac{1}{\sqrt{3}} \sqrt{1-\left|\omega_{1}\right|^{2}} \\
& +2 \operatorname{Re}\left\{(5 / 3+4 q+3 p) \omega_{1}^{3}-\omega_{1} l^{2}\right\} .
\end{aligned}
$$

We write $\omega_{1}=x e^{i \varphi}, 0 \leq x \leq 1$, and put $l=(2+p) x e^{-i \varphi / 2} \cos (3 \varphi / 2)$ and $y=|\sin (3 \varphi / 2)|, 0 \leq y \leq 1$. Then $\left|(2+p) \omega_{1}-l\right|=|2+p| x y$ and

$$
\begin{aligned}
\left|D_{4}\right| \leq & 2 / 3+2 b^{2} x^{2}\left(1-y^{2}\right)+4|b| x y \frac{\sqrt{1-x^{2}}}{\sqrt{3}} \\
& +2\left(a-b^{2}\right) x^{3}+2 y^{2}\left(b^{2}-2 a\right) x^{3}=\varphi(x, y),
\end{aligned}
$$

where $a=5 / 3+4 q+3 p$ and $b=2+p$.

1. The case $q \leq-3 p / 4-5 / 12$. Evidently, if $a=b=0$, then $D_{4} \leq 2 / 3$, so we omit this case. If $b=0$ and $a<0$, then the coefficient $z=\left(\left(b^{2}-\right.\right.$ $\left.2 a) x-b^{2}\right) x^{2}$ of $y^{2}$ in (3) is positive for all $0 \leq x \leq 1$. Analogously, if $b \neq 0$ and $a=0$, then $z \leq 0$ for all $0 \leq x \leq 1$. Let $x_{0}=b^{2} /\left(b^{2}-2 a\right)$; then $x_{0} \in[0,1]$. If $a \neq 0$ and $b \neq 0$, then $0<x_{0}<1$. Let $x_{0} \leq x \leq 1$. Hence $z \geq 0$ and $\max _{0 \leq y \leq 1} \varphi(x, y)=\varphi(x, 1)$. If $0<x<x_{0}$, then $z<0$ and $\max _{0 \leq y \leq 1} \varphi(x, y)=\varphi\left(x, y^{*}\right)$, where

$$
y^{*}=\frac{|b| \sqrt{1-x^{2}}}{x \sqrt{3}\left[b^{2}(1-x)+2 a x\right]} .
$$

Elementary calculations show that the inequality $|p+2|<2 \sqrt{2} / \sqrt{3}$ implies that $y^{*}>1$ is equivalent to $|p+2|<2 \sqrt{2} / \sqrt{3}$. Thus, if $|b|<2 \sqrt{2} / \sqrt{3}$ and $a<0$, then

$$
\left|D_{4}\right| \leq 2 / 3-2 a x^{3}+\frac{4|b|}{\sqrt{3}} x \sqrt{1-x^{2}}=2 \Phi_{1}(x)+2 / 3,
$$

and $\Phi_{1}(0)=0, \Phi_{1}(1)>0$. It is not difficult to show that $\Phi_{1}(x)$ has a unique maximum in $(0,1)$ at the point $x^{*}$, where $x^{*}$ is the unique root in $(0,1)$ of the equation

$$
3 a x^{2} \sqrt{1-x^{2}}+\frac{4|b|}{\sqrt{3}} x^{2}-\frac{2|b|}{\sqrt{3}}=0 .
$$

Note that if $x=x_{0}$, then $\varphi(x, y)$ is a linear function of $y$ and $\max _{0 \leq y \leq 1} \varphi(x, y)$ $=\varphi(x, 1)$. If $x=0$, then evidently $y^{*}>1$.

Now, if $a=0$ and $b \neq 0$, then $z \leq 0, x \in[0,1]$ and

$$
y^{* 2}=(1+x) /\left(3 x^{2} b^{2}(1-x)\right) .
$$

Clearing up the inequality $y^{*}>1$ we come to

$$
\max _{0 \leq y \leq 1} \varphi(x, y)=\varphi(x, 1), \quad \max _{0 \leq x \leq 1} \Phi_{1}(x)=\Phi_{1}(1 / \sqrt{2}) .
$$


If $a \neq 0$ and $b=0$, then the maximum (4) holds again and $\left|D_{4}\right| \leq$ $8 / 3+8 q+6 p$.

Theorem 1. If $q \leq-3 p / 4-5 / 12$ and $|p+2| \leq 2 \sqrt{2 / 3}$, then

$$
\left|D_{4}\right| \leq 2 / 3-2(5 / 3+4 q+3 p) x^{* 3}+\frac{4|p+2|}{\sqrt{3}} x^{*} \sqrt{1-x^{* 2}},
$$

where $x^{*}$ is the unique root in $(0,1)$ of the equation

$$
\sqrt{3}(5+12 q+9 p) x^{2} \sqrt{1-x^{2}}+|4+2 p|\left(2 x^{2}-1\right)=0 .
$$

The inequality (5) is sharp only for $p=-2$ and $q=13 / 12$.

Corollary. If $f(z)=z+\sum_{n=2}^{\infty} c_{n} z^{n} \in \mathbb{S}$, then

$$
\left|c_{4}-2 c_{2} c_{3}+q c_{2}^{3}\right| \leq \begin{cases}8(q-1) & \text { for } q \geq 13 / 12, \\ (4 / 3)(7-6 q) & \text { for } q<13 / 12,\end{cases}
$$

2) $\quad\left|c_{4}+p c_{2} c_{3}-(3 p / 4+5 / 12) c_{2}^{3}\right| \leq 2 / 3+2|p+2| / \sqrt{3}$

$$
\text { if }|p+2| \leq 2 \sqrt{2 / 3} \text {. }
$$

Now we consider the case $|p+2|>2 \sqrt{2 / 3}$. We want $y^{*}$ to be bigger than 1 again. This condition implies the inequality

$$
\Psi(x)=3 x^{2} b^{4}+6 x^{3} b^{2}\left(2 a-b^{2}\right)+3 x^{4}\left(2 a-b^{2}\right)^{2}-b^{2}\left(1-x^{2}\right)<0 .
$$

To prove this, the sign of $\Psi^{\prime}(x)$ can be determined or

$$
u(x)=\frac{\Psi^{\prime}(x)}{2 x}=6 x^{2}\left(b^{2}-2 a\right)^{2}-9 b^{2} x\left(b^{2}-2 a\right)+b^{2}\left(1+3 b^{2}\right)>0 .
$$

From $\Psi\left(x_{0}\right)=\left(4 b^{4} a-4 a^{2} b^{2}\right)\left(b^{2}-2 a\right)^{-2}<0$ it will follow that $\Psi(x)<0$. The equation $u(x)=0$ has two real roots

$$
x_{1,2}=\frac{9 b^{2} \pm b \sqrt{3\left(3 b^{2}-8\right)}}{12\left(b^{2}-2 a\right)} .
$$

We put $b>0, x_{1}<x_{2}$. So $x_{2}<x_{0}$. We want $\Psi\left(x_{1}\right)$ to be negative. To simplify the form of the corresponding curve in the $(p, q)$-plane we find that the inequality

$$
q<-\frac{9}{128} p^{4}-\frac{9}{16} p^{3}-\frac{209}{128} p^{2}-\frac{89}{32} p-\frac{127}{96}=A(p)
$$

implies $\Psi\left(x_{1}\right)<0$. Hence if $q<A(p)$, then $y^{*}>1$, and the considerations of Theorem 1 remain true, so $\max _{0 \leq y \leq 1} \varphi(x, y)=\varphi(x, 1)$. Obviously, if $q<A(p)$, then $q \leq-3 p / 4-5 / 12$.

Theorem 2. If $|p+2|>2 \sqrt{2 / 3}$ and

$$
q<-\frac{9}{128} p^{4}-\frac{9}{16} p^{3}-\frac{209}{128} p^{2}-\frac{89}{32} p-\frac{127}{96},
$$

then the estimate (5) holds. 
2. The case $q>-3 p / 4-5 / 12$. The coefficient $z=x^{2}\left(-2 a x-b^{2}(1-x)\right)$ of $y^{2}$ in $\varphi(x, y)$ is negative, and $\varphi(x, y) \leq \varphi\left(x, y^{*}\right)$, where

$$
y^{*}=\frac{|b| \sqrt{1-x^{2}}}{\sqrt{3} x\left(b^{2}-\left(b^{2}-2 a\right) x\right)}
$$

and

$$
\begin{aligned}
\left|D_{4}\right| & \leq 2 / 3+2 b^{2} x^{2}+2\left(a-b^{2}\right) x^{3}-\frac{2 b^{2}\left(1-x^{2}\right)}{3\left(\left(b^{2}-2 a\right) x-b^{2}\right)} \\
& =2 / 3+2 \Phi_{2}(x) .
\end{aligned}
$$

Now we look for the region of the $(p, q)$-plane where the Koebe function is extremal. In this case we should have the inequality $\Phi_{2}(x) \leq \Phi_{2}(1)$. We assume

$$
\begin{aligned}
& a \leq b^{2} \leq 2 a, \\
& b^{2}<a(9-\sqrt{17}) / 4, \\
& b^{2}<6 a^{2}, \\
& a \geq 1 / 3 .
\end{aligned}
$$

We reduce the inequality $\Phi_{2}(x) \leq \Phi_{2}(1)$ to a common denominator taking into account (6) and get it in the equivalent form:

$\Psi(x)=3\left(b^{2}+\left(2 a-b^{2}\right) x\right)\left\{b^{2}(1+x)+\left(a-b^{2}\right)\left(1+x+x^{2}\right)\right\}-b^{2}(1+x) \geq 0$.

The inequality (6) implies that $\Psi^{\prime \prime}(x)$ is decreasing and since by (7), $\Psi^{\prime \prime}(1) / 6=8 a^{2}-9 a b^{2}+2 b^{4}>0$ it follows that $\Psi^{\prime \prime}(x)>0$ for $x \in[0,1]$. $\Psi^{\prime}$ increases and by $(8), \Psi^{\prime}(0)=6 a^{2}-b^{2}>0$. Hence, $\Psi(x)$ increases and by $(9), \Psi(0)=b^{2}(3 a-1) \geq 0$ and therefore $\Psi(x) \geq 0$ for all $x \in[0,1]$.

Now we consider the region containing the point $(0,0)$ of the $(p, q)$-plane. In the case $a \geq 0, a \leq b^{2} / 2$ we make $\Phi_{2}(x)$ bigger, so

$$
\Phi_{2}(x) \leq b^{2} x^{2}+\left(a-b^{2}\right) x^{3}+b^{2}\left(1-x^{2}\right) /(6 a)=g(x) .
$$

If

$$
q>p^{2} / 24-7 p / 12-1 / 4+\frac{|p+2|}{24}\left((p+2)^{2}+4\right)^{1 / 2},
$$

then $b^{2}<9 a^{2} /(1+3 a), g^{\prime}(x)>0$ for $x \in[0,1]$ and

$$
\left|D_{4}\right| \leq 2 / 3+2 \Phi_{2}(x) \leq 2 / 3+2 g(x) \leq 2 / 3+2 g(1)=2 / 3+2 \Phi_{2}(1) .
$$

Hence, here the Koebe function is also extremal.

Theorem 3. If

$$
\frac{1}{12(9-\sqrt{17})}\left(12 p^{2}+5(3 \sqrt{17}-11) p+5 \sqrt{17}+3\right) \leq q \leq p^{2} / 4+p / 4+7 / 12
$$


and $q>-3 p / 4-1 / 3$, or if

$$
p^{2} / 24-7 p / 12-1 / 4+\frac{|p+2|}{24}\left((p+2)^{2}+4\right)^{1 / 2}<q<p^{2} / 8-p / 4+1 / 12,
$$

and $1+4 q+3 p>0$, then $\left|D_{4}\right| \leq 4+6 p+8 q$ with the Koebe function being extremal (the point $(0,0)$ belongs to the last domain).

\section{References}

[1] Z. J. Jakubowski, H. Siejka and O. Tammi, On the maximum of $a_{4}-3 a_{2} a_{3}+\mu a_{2}$ and some related functionals for bounded real univalent functions, Ann. Polon. Math. 46 (1985), 115-128.

[2] J. Ławrynowicz and O. Tammi, On estimating of a fourth order functional for bounded univalent functions, Ann. Acad. Sci. Fenn. Ser. AI 490 (1971), 1-18.

[3] N. A. Lebedev, Area Principle in the Theory of Univalent Functions, Nauka, Moscow, 1975 (in Russian).

[4] P. Lehto, On fourth-order homogeneous functionals in the class of bounded univalent functions, Ann. Acad. Sci. Fenn. Ser. AI Math. Dissertationes 48 (1984).

[5] K. Włodarczyk, On certain non-homogeneous combinations of coefficients of bounded univalent functions, Demonstratio Math. 16 (1983), 919-924.

DEPARTMENT OF PHYSICS\&MATH. SARATOV PEDAGOGICAL INSTITUTE 92 MICHURIN ST

SARATOV 410071, RUSSIA

\begin{abstract}
DEPARTMENT OF MATH.\&MECH. SARATOV STATE UNIVERSITY 83 ASTRAKHANSKAYA ST.
\end{abstract} SARATOV 410071, RUSSIA 